\title{
Fast Rotation-Invariant Video Caption Detection Based on Visual Rhythm
}

\author{
Felipe Braunger Valio, Helio Pedrini, and Neucimar Jeronimo Leite \\ Institute of Computing - University of Campinas \\ Campinas, SP, Brazil, 13084-971
}

\begin{abstract}
Text detection in images has been studied and improved for decades. There are many works that extend the existing methods for analyzing videos, however, few of them create or adapt approaches that consider inherent characteristics of videos, such as temporal information. This work proposes a very fast method for identifying video frames that contain text through a special data structure called visual rhythm. The method is robust to detect video captions with respect to font styles, color intensity, and text orientation. A data set was built in our experiments to compare and evaluate the effectiveness of the proposed method.
\end{abstract}

\section{Introduction}

Several video-based applications are becoming more common, driven by factors such as popularization of digital television, increase of bandwidth for data transmission via Internet, evolution of augmented reality research, and the development of mobile equipments for multimedia purpose.

Texts in video provide relevant information of a scene content. For instance, a portion of the video is commonly used in news to exhibit information such as weather forecast, sport scores, text alerts, financial market overview. Captions are employed in TV documentaries to describe a location, a person, a title or an event. Almost all commercials use a certain type of subtitle to provide more information of products, since their exhibition time is restricted to only few seconds. In movies, subtitles are used to inform their cast and credits, or an introductory story. Systems have also become more practical for text translation, navigation based on textual content, indexing of multimedia libraries, detection of events such as appearance of commercials, blocks of news, among others.

Since videos concentrate a large amount of information, their analysis is a task of high computational cost. A way to speed up the caption detection process is to eliminate frames that certainly are not subtitled.

This paper describes and evaluates a fast method for detecting video frames containing captions based on a data structure called visual rhythm. The captions can be written in different languages and orientations. The visual rhythm can be generated in multiple scales by means of a space-filling curve. Regions of interest are segmented from the visual rhythm and then classified as captions or non-captions according to a small number of rules. 
The text is organized as follows. Section 2 describes some related work available in the literature. The definitions of visual rhythm and video caption are introduced in Sections 3 and 4, respectively. In Section 5, the proposed method is presented and discussed. Experimental results obtained are shown in Section 6. Finally, Section 7 concludes with some final remarks.

\section{Related Work}

Most works related to caption detection are based on approaches used to identify texts in still images, where temporal information is also considered.

Some works consider the pre-calculated MPEG DCT coefficients to speed up the detection process. The work in 2] performs the closed caption detection in sports videos by filtering group of images not supposed to have subtitles. In [12, DCT coefficients are taken into account to extract contrast and regularity information, which can be used to detect the existence of captions in video frames. Both considered only the I-frames in MPEG video streams. The work in 13 also detects captions on the I-frames of a MPEG sequence. In this case, the DCT coefficients are filtered based on texture analysis. These three strategies fail to detect the exact frames from the beginning and end of the subtitle.

The work in 5] considers an SVM classifier in the discrimination of features derived from a wavelet transform. The frame rate is reduced to $1 \mathrm{fps}$ to speed up the method, so it cannot precisely define the range of frames conveying the corresponding caption information.

A set of morphological operators is used in [10] to detect candidate regions in each frame. Further, statistical moment and linear projections are considered in the textual classification of the image components. In order to emphasize contrast, a convolution filter is introduced in [1], followed by the analysis of edge density and connected components in each sub-region of a frame. Both works perform a frame-by-frame detection, showing that they are costly operations.

In 3], the authors consider the visual rhythm to locate potential frames with subtitles. A Prewitt filter is used to extract horizontal edges. Each edge, after a size filtering, is considered a potential caption. This strategy proved to generate many false positives. The used visual rhythm corresponds to the union of the vertical, diagonal, and reverse diagonal lines.

Finally, the work in [6] analyzes an initial set of frames and try to refine the obtained results, for the next frames, through the assumption that the previously detected captions should last for a certain period of time.

\section{Visual Rhythm}

A video slice is defined as a set of pixels of an image linearly arranged in a $1 \mathrm{D}$ signal. The visual rhythm is then represented by the slices of all frames of a video sequence, kept together in a certain manner. Equation 1 defines a 1D slice, $f$, of a frame $F$ with $M$ rows and $N$ columns. 


$$
f[i]=\sum_{p=k-j}^{k+j} \alpha_{p} F(i, p)
$$

where $0 \leq i<M, 0 \leq p<M, k=M / 2$, and $\sum \alpha_{p}=1$. When $j=0$, pixels in the middle column of $F$ are taken to form the corresponding slice. Here, to guarantee a certain contrast homogeneity of the slices we consider $j=1$ and perform a maximum filter in Equation 1, such that

$$
f[i]=\max \left[\alpha_{p-1} F(i, p-1), \alpha_{p} F(i, p), \alpha_{p+1} F(i, p+1)\right]
$$

By putting these slides together over time, we define a 2D image which constitutes the visual rhythm representation [8]. Independently from the way the slices are formed, each slice is disposed vertically to generate the corresponding visual rhythm whose width depends on the number of frames in the whole video.

Figure 1 shows the visual rhythm for a sequence of captioned frames in a video. Several features can be clearly observed from the figure, such as scene transitions. The captions, in particular, are easily identifiable, represented by the white rectangular bands in the lower image area.

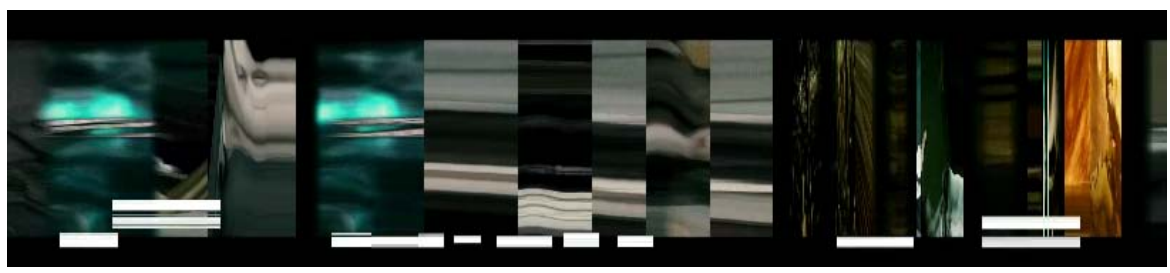

Fig. 1. Visual rhythm generated from captioned video frames

To improve the caption detection process, more detailed curves can be used to extract pixels more evenly distributed along the frames compared to a vertical slice, as defined in Equation 1. Although the use of space-filling curves [9, such as Hilbert, Peano, or zig-zag scans, increases the visual rhythm height and, consequently, execution time, the results of the caption detection process are more precise, as reported in Section 6 .

\section{Caption Features}

In this work, we consider the following basic aspects of the subtitled texts in a video sequence: (i) they are superimposed to the images by an editing technique, and (ii) there is no caption motion during a certain range of time.

The work in 4 describes important features related to subtitling practices in image sequences, which include duration and height of captions. Some of these issues are taken into account in our work to establish some caption filters. One 
of them considers that, except for extreme cases, the lowest line of the caption should be at least 1/12 of the screen height just above the bottom of the screen.

According to the aforementioned work, the minimum duration of a single-word subtitle should be $3 / 2$ seconds. For the spectator to clearly perceive a caption transition, at least $1 / 4$ of a second needs to be inserted between two consecutive subtitles.

\section{Proposed Methodology}

The main steps of the proposed methodology are illustrated in Figure 2, which are described in more details in the following subsections.

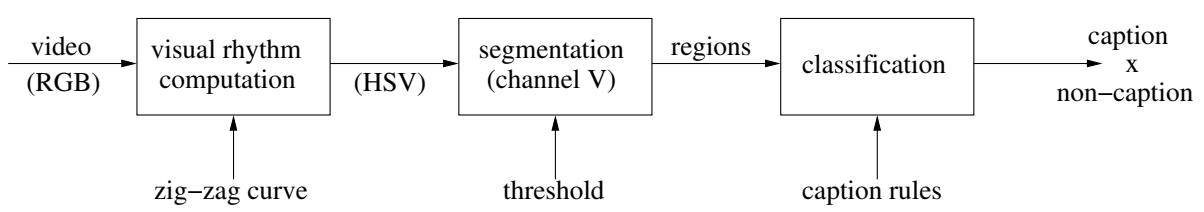

Fig. 2. Diagram of the proposed method

\subsection{Visual Rhythm Calculation}

Initially, each frame of the video sequence, in RGB format, is scanned by a certain curve to produce a slice. The zig-zag curve, shown in Figure 3. was chosen since it is simple and capable of passing across captions in arbitrary orientations. This curve can be parameterized at multiple scales by varying its number of diagonal lines. The greater the curve length, the larger the size of the visual rhythm, such that a proper scale should be obtained to balance efficiency and accuracy. Results are shown in Section 6.

To avoid a caption not being detected during the curve traversal by passing, for instance, exactly between two words, a morphological dilation operation is applied to each color band of the original frame. A way of speeding up this process is to apply the dilation only to pixels passing through the curve. Each frame can also be partitioned into a number of blocks with uniform size. For

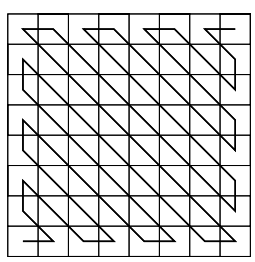

(a)

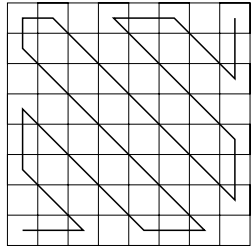

(b)

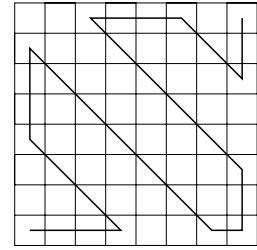

(c)

Fig. 3. Examples of zig-zag curves in three different scales 
instance, when frames are divided into $5 \times 5$ blocks, coarser or finer scale curves could be used in each block to produce portions of slices. The resulting visual rhythm is obtained by the union of these 25 partial slices and converted into HSV color space.

The proposed segmentation and classification approaches are described in the following subsections, as shown in Figure 2. These two steps should be quite simple in order to improve computational time.

\subsection{Segmentation}

The visual rhythm segmentation is basically the extraction of candidate caption regions in the $\mathrm{V}$ channel of HSV format. A threshold is applied to the visual rhythm image to separate the regions from the background. It is worth mentioning that captions are embedded into the video frames, assuring a proper contrast with the foreground.

As a result of the segmentation process, connected regions are formed by a set of pixels with low gradient. Figure 4 gives an example of segmented image from a portion of the visual rhythm shown in Figure 1.

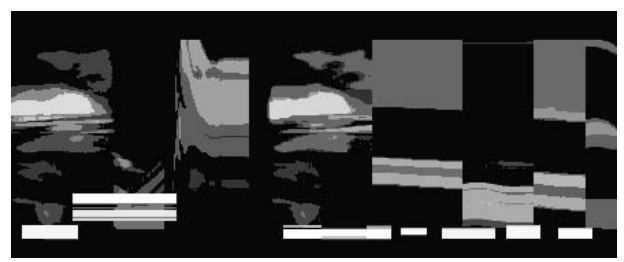

Fig. 4. Result of segmentation process for a portion of the visual rhythm shown in Figure 1

\subsection{Classification}

The classification step labels the segmented regions as caption or non-captions. As mentioned before, captions will form rectangular areas. Ideally, each caption in the visual rhythm generates a single connected region after the segmentation.

The classification has three steps. Initially, regions that do not meet certain requirements for caption dimensions are discarded. Captions should be at least 45 pixels wide and at most 1/12 of the frame height, as discussed in Section 4 . Second, a measure of rectangularity, defined as the ratio between the area of a segmented region and the area of its bounding box, should be within a tolerance value. Third, a thinning algorithm [11] is applied to each region, which will generate a horizontal line if the region is a rectangle. Regions that do not form horizontal lines are discarded. 


\section{Results}

Results for a number of video sequences are reported in this section. The data set is formed by a collection of videos of various categories, containing captions in different languages (western, eastern and middle eastern) and orientations. Due to the difficulty in finding videos with captions in any orientation, subtitles rotated by $10^{\circ}$ and $20^{\circ}$ were embedded into some videos. The beginning and end frame numbers in which captions appear in the tested videos were manually identified to be used as ground truth. All videos are in a resolution of about $480 \times 270$ pixels. The data set used in our experiments is available in 7 . The method has been implemented in $\mathrm{C}++$ on a PC with $2.3 \mathrm{GHz}$ Pentium IV CPU and 1GB RAM memory.

The visual rhythm was adequately generated by using a zig-zag curve with three lines. Each frame can be partitioned into $3 \times 3$ regions. A threshold value of 40 was used to separate the candidate caption regions, whereas a rectangularity measure of 0.95 was used to classify the regions as captions or non-captions.

The proposed methodology was applied to the video sequences and compared against three different approaches. The F-measure, computed through precision and recall rates, as well as the number of frames per second during the execution, are used to evaluate the performance of our method. The F-measure is defined as

$$
\text { F-Measure }=2 * \frac{\text { precision } * \text { recall }}{\text { precision }+ \text { recall }}
$$

where precision $=\frac{t p}{t p+f p}$, recall $=\frac{t p}{t p+f n}$, and $f p$, fn and tp correspond to false positives, false negatives and true positives, respectively.

Table 1 presents the results obtained with the tested methods. Even with simple segmentation and classification approaches, it is possible to see that the proposed method produces superior results for the majority of the video sequences, including captions written in Japanese and Arabic languages, as well as rotated captions.

Table2 2 shows the average number of video frames analyzed per second (FPS). It can be observed that the use of visual rhythm is much faster than the frame-byframe approaches. Although the proposed method slightly looses in performance when compared with [3, it is possible to speed up the process in detriment of certain accuracy level.

The plot, shown in Figure 5 presents the F-measure for the proposed method, varying the number of frame partitions $(1 \times 1,3 \times 3$ or $5 \times 5$ blocks $)$ and the number of diagonal lines $(1,3,5$ or 7$)$ used to build the zig-zag curve. It can be observed that it is not advantageous to divide the video frames into $5 \times 5$ blocks. The best result was obtained when no fragmentation $(1 \times 1)$ was applied, however, this requires a more detailed curve ( 5 lines), costing more processing time. On the other hand, the video fragmentation into $3 \times 3$ blocks and the use of 3 lines produce a nearly identical result, justifying the choice of this setting in our experiments. A very detailed curve (7 lines) has a significant drop in the plot due to the increase of the number of false positives. 
Table 1. Video data sets and performance of the tested approaches

\begin{tabular}{|l|l|r|r|r|r|r|r|}
\hline \multicolumn{2}{|c|}{} & \multicolumn{5}{|c|}{ Data Sets } \\
\hline Method & Measures (\%) & News & Movies & Commercial & Japanese & Arabic & Rotated \\
\hline \hline Proposed & Precision & 76.7 & 78.5 & 79.0 & 94.7 & 96.6 & 79.6 \\
& Recall & 97.6 & 93.8 & 100.0 & 99.7 & 95.8 & 99.6 \\
& F-Measure & $\mathbf{8 5 . 9}$ & 84.7 & $\mathbf{8 8 . 2}$ & $\mathbf{9 7 . 1}$ & $\mathbf{9 6 . 2}$ & $\mathbf{8 8 . 5}$ \\
\hline Wu et al. [10] & Precision & 73.5 & 77.4 & 61.4 & 88.0 & 69.0 & 65.4 \\
& Recall & 99.5 & 94.8 & 98.5 & 99.5 & 99.0 & 100.0 \\
& F-Measure & 84.6 & $\mathbf{8 5 . 0}$ & 75.7 & 93.4 & 81.3 & 79.1 \\
\hline Agnihotri and & Precision & 90.0 & 85.3 & 65.5 & 79.6 & 68.0 & 75.1 \\
Dimitrova [1] & Recall & 73.6 & 66.7 & 57.1 & 33.3 & 18.0 & 56.3 \\
& F-Measure & 81.0 & 74.8 & 61.0 & 46.9 & 28.5 & 64.3 \\
\hline Chun et al. [3] & Precision & 41.7 & 33.8 & 73.1 & 24.8 & 14.0 & 73.9 \\
& Recall & 99.8 & 88.3 & 92.9 & 94.1 & 92.1 & 60.4 \\
& F-Measure & 58.9 & 47.4 & 81.8 & 39.2 & 24.3 & 66.4 \\
\hline
\end{tabular}

Table 2. Average number of frames analyzed per second for each tested approach

\begin{tabular}{|l|r|}
\hline Method & FPS \\
\hline \hline Proposed & 93.10 \\
\hline Wu et al. [10] & 0.84 \\
\hline Agnihotri and Dimitrova [1] & 2.52 \\
\hline Chun et al. [3] & 109.24 \\
\hline
\end{tabular}

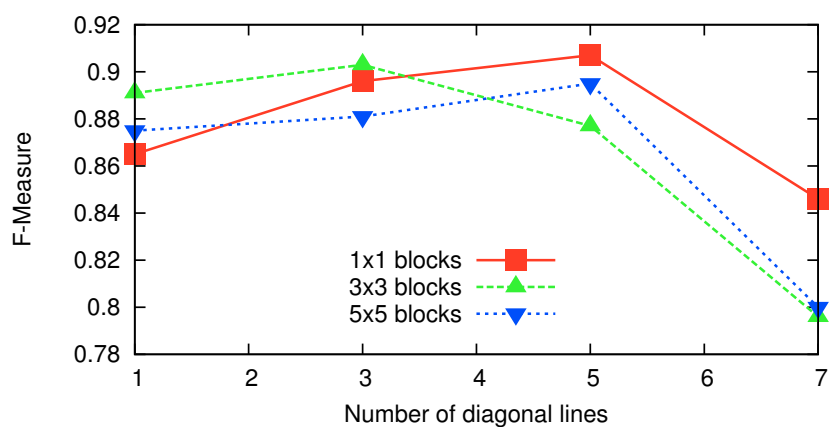

Fig. 5. Results for F-Measure obtained by varying the number of diagonal lines in the zig-zag curve and the number of frame blocks for the proposed method

\section{Conclusions and Future Work}

A new video caption detection method is described in this work. A data structure, called visual rhythm, is created by sub-sampling each video frame with a 
multi-scale curve. The captions are detected from the visual rhythm through a set of simple rules.

The method is robust to detect captions located in any position and orientation in the video frames, as well as unknown text layout common in different languages. Experimental results demonstrated the effectiveness of the proposed method compared to three other approaches.

As future work, we plan to investigate more effective techniques for segmenting and classifying the visual rhythm, which may significantly improve the results of the proposed method in terms of precision and recall rates.

Acknowledgments. The authors are thankful to FAPESP, CNPq and CAPES for the financial support.

\section{References}

1. Agnihotri, L., Dimitrova, N.: Text Detection for Video Analysis. In: IEEE Workshop on Content-Based Access of Image and Video Libraries, pp. 109-113 (1999)

2. Chen, D.Y., Hsiao, M.H., Lee, S.Y.: Automatic Closed Caption Detection and Font Size Differentiation in MPEG Video. In: Chang, S.-K., Chen, Z., Lee, S.-Y. (eds.) VISUAL 2002. LNCS, vol. 2314, pp. 276-287. Springer, Heidelberg (2002)

3. Chun, S.S., Hyeokman, K., Jung-Rim, K., Sangwook, O., Sanghoon, S.: Fast text caption localization on video using visual rhythm. In: Chang, S.-K., Chen, Z., Lee, S.-Y. (eds.) VISUAL 2002. LNCS, vol. 2314, pp. 259-268. Springer, Heidelberg (2002)

4. Karamitroglou, F.: A Proposed Set of Subtitling Standards in Europe. Translation Journal 2(2) (February 2007)

5. Lee, C.C., Chiang, Y.C., Huang, H.M., Tsai, C.L.: A Fast Caption Localization and Detection for News Videos. In: International Conference on Innovative Computing, Information and Control, Los Alamitos, CA, USA, pp. 226-229 (September 2007)

6. Lienhart, R., Wernicke, A.: Localizing and Segmenting Text in Images and Videos. IEEE Transactions on Circuits and Systems for Video Technology 12(4), 256-268 (2002)

7. LIV: Video Sequences (2010), http://www.liv.ic.unicamp.br/ felipebvalio/base.zip

8. Ngo, C., Pong, T., Chin, R.: Detection of Gradual Transitions through Temporal Slice Analysis. In: IEEE Conference on Computer Vision and Pattern Recognition, pp. 36-41 (1999)

9. Sagan, H.: Space-Filling Curves. Springer, New York (1994)

10. Wu, J.-C., Hsieh, J.-W., Chen, Y.-S.: Morphology-based Text Line Extraction. Machine Vision and Applications 19(3), 195-207 (2008)

11. Zhang, T.Y., Suen, C.Y.: A fast parallel algorithm for thinning digital patterns. Communications of the ACM 27, 236-239 (1984)

12. Zhang, Y., Chua, T.S.: Detection of Text Captions in Compressed Domain Video. In: ACM Multimedia, Los Angeles, CA, USA, pp. 201-204 (2000)

13. Zhong, Y., Zhang, H., Jain, A.: Automatic Caption Localization in Compressed Video. IEEE Transactions on Pattern Analysis and Machine Intelligence 22(4), 385-392 (2000) 